\title{
Effects of imidazoline-like drugs on liver and adipose tissues, and their role in preventing obesity and associated cardio-metabolic disorders
}

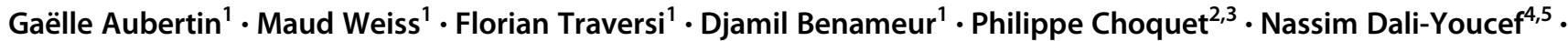 \\ Françoise Pons ${ }^{6} \cdot$ Séverine Sigrist $^{7} \cdot$ Hugues Greney ${ }^{1} \cdot$ Laurent Monassier $^{1} \cdot$ Pascal Bousquet $^{1}$. \\ Nathalie Niederhoffer ${ }^{1}$
}

Received: 9 July 2018 / Revised: 31 January 2019 / Accepted: 10 March 2019

(c) Springer Nature Limited 2019

\begin{abstract}
Background/Objectives We previously observed that selective agonists of the sympatho-inhibitory $\mathrm{I}_{1}$ imidazoline receptors (LNP ligands) have favorable effects on several cardiovascular and metabolic disorders defining the metabolic syndrome, including body weight. The objectives of this study were to explore the effects of LNPs on adiposity and the mechanisms involved, and to evaluate their impact on metabolic homeostasis.

Methods Young Zucker fa/fa rats were treated with LNP599 $(10 \mathrm{mg} / \mathrm{kg} /$ day $)$ for 12 weeks. Effects on body weight, adiposity (regional re-distribution, morphology, and function of adipose tissues), cardiovascular and metabolic homeostasis, and liver function were evaluated. Direct effects on insulin and AMP-activated protein kinase (AMPK) signaling were studied in human hepatoma HepG2 cells.

Results LNP599 treatment limited the age-dependent remodeling and inflammation of subcutaneous, epididymal, and visceral adipose tissues, and prevented total fat deposits and the development of obesity. Body-weight stabilization was not related to reduced food intake but rather to enhanced energy expenditure and thermogenesis. Cardiovascular and metabolic parameters were also improved and were significantly correlated with body weight but not with plasma norepinephrine. Insulin and AMPK signaling were enhanced in hepatic tissues of treated animals, whereas blood markers of hepatic disease and pro-inflammatory cytokine levels were reduced. In cultured HepG2 cells, LNP ligands phosphorylated AMPK and the downstream acetyl-CoA carboxylase and prevented oleic acid-induced intracellular lipid accumulation. They also significantly potentiated insulin-mediated AKT activation and this was independent from AMPK.

Conclusions Selective $\mathrm{I}_{1}$ imidazoline receptor agonists protect against the development of adiposity and obesity, and the associated cardio-metabolic disorders. Activation of $\mathrm{I}_{1}$ receptors in the liver, leading to stimulation of the cellular energy sensor AMPK and insulin sensitization, and in adipose tissues, leading to improvement of morphology and function, are identified as peripheral mechanisms involved in the beneficial actions of these ligands.
\end{abstract}

These authors contributed equally: Gaëlle Aubertin and Maud Weiss

Supplementary information The online version of this article (https:// doi.org/10.1038/s41366-019-0342-z) contains Supplementary

Material, which is available to authorized users.

Nathalie Niederhoffer

nathalie.niederhoffer@unistra.fr

Extended author information available on the last page of the article

\section{Introduction}

Metabolic syndrome (MetS) is clinically defined as a cluster of interrelated risk factors, among which hypertension, obesity, dyslipidemia, and insulin resistance $[1,2]$, which promote the risk for cardiovascular and metabolic diseases (atherosclerosis, type 2 diabetes, etc.), and largely increase morbi-mortality [3].

Over the last decade, chronic hyperactivity of the sympathetic nervous system has emerged as one of the key pathophysiological factors of the MetS [4-6]. As a consequence, sympatho-inhibition has been proposed as a possible strategy for the prevention and/or treatment of this 
syndrome. In agreement, some studies demonstrated that reducing sympathetic nervous system activity by renal sympathetic denervation is efficient not only to reduce blood pressure but also to improve glucose homeostasis and insulin sensitivity [7-9]. Such non-pharmacological procedure is however restricted to patients with uncontrolled resistant hypertension.

Sympatho-inhibition can be pharmacologically achieved by targeting medullary $I_{1}$ imidazoline receptors $\left(I_{1} R\right)$, which, together with $\alpha_{2}$-adrenergic receptors, are activated by centrally acting anti-hypertensive drugs [10]. We previously reported that original $\mathrm{I}_{1} \mathrm{R}$ selective ligands [LNP509 and LNP599 [11-13]] have favorable effects on insulin action, glucose tolerance, and lipid profile in a rat model of MetS (spontaneously hypertensive, heart failure rats (SHHF) rats) [14] and in spontaneous age-related insulin resistance [15]. Unexpectedly, LNP ligands (LNPs) not only reduced sympathetic tone by a central mechanism but also activated peripheral $I_{1} R$ to promote adiponectin synthesis and secretion from adipocytes and AMP-activated protein kinase (AMPK) signaling in hepatocytes [15]. As both adiponectin and AMPK (i) act as major insulin sensitizers, (ii) have a key role in the maintenance of glucose and lipid homeostasis, and (iii) are dysregulated in MetS [16-20], these additional peripheral effects probably contribute to the overall beneficial action of LNPs on MetS symptoms.

Insulin resistance and metabolic disorders are closely linked to overweight, which has been retained by the International Diabetes Federation (IDF) as a mandatory criterion for the diagnosis of MetS [1]. Obesity is characterized by morphological and functional changes in adipose tissue (AT), with hypertrophic adipocytes and macrophage infiltration, leading to altered secretory profile of adipokines, and exaggerated production and release of free fatty acids and pro-inflammatory cytokines, which all have been involved in the development of insulin resistance (for recent reviews, see, e.g., refs. [21, 22]) and are good markers of MetS [23]. Strikingly, we also observed that the body weight of rats chronically treated with LNP599 tended to stabilize, whereas it continued to increase in control rats [14]. The mechanism(s) underlying this effect, as well as its "clinical" relevance (regional re-distribution, morphology and function of ATs, impact on peripheral insulin resistance, and metabolic homeostasis) remained unknown.

Therefore, the objective of the present study was to further explore and understand the effects of $\mathrm{I}_{1} \mathrm{R}$ ligands on adiposity, insulin sensitivity, and glucose and lipid regulations. To this end, young Zucker rats were treated for 12 weeks with LNP599. In these rats, leptin signaling is altered, leading to hyperphagia and obesity. Functionally, Zucker rats exhibit moderate hypertension, insulin resistance without fasting hyperglycemia, and hyperlipidemia, and as such may represent the rat model that fits most the IDF criteria of the MetS. In addition, hypersympathicotony is much less pronounced in this rat strain than in the SHHF rats used previously; we therefore expected that LNP599 effects independent from sympatho-inhibition would be predominent. Direct hepatic effects were also studied in cultured human hepatoma HepG2 cells. Studies were designed in order to explore (i) the extent to which the effects of LNP599 on insulin resistance observed in vivo could be due to a direct effect on hepatocytes and (ii) the functional consequences of LNP599-induced AMPK activation on insulin sensitivity and lipid accumulation.

\section{Methods}

\section{Animals and LNP599 treatment}

Twenty-three 12-week-old male Zucker fa/fa rats (Charles River Laboratories, L'Arbresle, France) were randomized to receive or not LNP599 (3-chloro-2-methyl-phenyl)-(4methyl-4,5-dihydro-3H-pyrrol-2-yl)-amine hydrochloride) [12] at the dose of $10 \mathrm{mg} / \mathrm{kg} /$ day over 12 weeks (LNP599 group, $n=12$; control group, $n=11$ ). In treated animals, LNP599 was delivered continuously $(2.5 \mu \mathrm{L} / \mathrm{h})$ by subcutaneous osmotic pumps (Alzet 2ML4) implanted under isoflurane $(1.5-2.5 \%)$ anesthesia at the beginning of the treatment (T0) and then replaced every 4 weeks (at T4 and T8). Control animals were sham-operated at the same time points. Previous studies confirmed that this dose of LNP599 is devoid of acute and chronic toxicity (data not shown; see also ref. [14]). During the whole study, animals were housed in a temperature- and light-controlled room with free access to standard diet (A04; SAFE, Augy, France), except for $12 \mathrm{~h}$ fasting periods before blood sampling and metabolic phenotyping; water was always available ad libitum. All procedures conformed to the French and European guidelines and regulations, and were approved by the Regional Ethics Committee for Animal Experimentation of Strasbourg (CREMEAS \#AL/12/41/12/12).

Baseline (T0) cardiovascular and metabolic parameters of Zucker rats were compared with values obtained in agematched male Sprague-Dawley rats $(n=11$; Elevage Janvier, St. Berthevin, France) (Supplementary Fig. 1); parameters were then evaluated every 4 weeks and/or at the end of the treatment period. Blood was sampled at the same time points (i.e., T0, T4, T8, and T12) from the tail vein of isoflurane-anesthetized rats. After a $15 \mathrm{~min}$ centrifugation at $2000 \times g$, the plasma was sampled and frozen at $-80{ }^{\circ} \mathrm{C}$ until assays. Biopsies from subcutaneous ATs (SCATs) and epididymal ATs (EATs) were taken at T0 and T8, immediately after blood sampling. Body weight, body temperature (measured at the skin surface at the interscapular level, 
i.e., above brown AT (BAT); ThermoFlash LX-26 Evolution), and water and food intakes were monitored twice a week. Night/day distribution of food intake was evaluated at $\mathrm{T} 0$ and $\mathrm{T} 12$ by measuring food consumption every $2 \mathrm{~h}$ over $24 \mathrm{~h}$. At the end of the in vivo experiments, rats were administered an intravenous (i.v.) lethal dose of pentobarbital; the ATs, pancreas, and liver were removed, weighed, and fixed in $4 \%$ paraformaldehyde for histology and immunostaining, or flash-frozen and stored at $-80{ }^{\circ} \mathrm{C}$ for western blotting analysis or enzyme-linked immunosorbent assays (ELISAa).

\section{Cardiovascular parameters}

Systolic blood pressure was measured in conscious rats using tail-cuff plethysmography (Bioseb Instruments, Vitrolles, France). Plasma concentrations of norepinephrine were measured by high-performance liquid chromatography (Plateau technique de Biochimie, University Hospital Strasbourg, France).

\section{Biochemical measurements}

Glucose levels were evaluated in a blood drop obtained from the tail vein using a glucometer (Accu Check, Roche Diagnostics, Meylan, France). Plasma insulin and adiponectin were quantified by ELISA kits (insulin: Mercodia, Uppsala, Sweden; adiponectin: Euromedex, Souffelweyersheim, France) according to the manufacturer's instructions. Homeostatic Model Assessment (HOMA) index of insulin resistance (IR) was calculated as fasting insulin $(\mathrm{mU} / \mathrm{L}) \times$ fasting glucose $(\mathrm{mmol} / \mathrm{L}) / 22.5$. Plasma total cholesterol (Cs), triglycerides (TGs), aspartate-alanine aminotransferases, and alkaline phosphatase were assayed using colorimetric kits (Euromedex, Souffelweyersheim, France). Cytokines (tumor necrosis factor- $\alpha$, interleukin- 6 , and the monocyte chemoattractant protein-1) in plasma and hepatic lysates were evaluated with ELISA cytokine profiling strips (Euromedex, Souffelweyersheim, France); data were expressed relative to the mean values of the control group.

\section{Measurement of fat mass by X-ray micro-computed tomography}

Total AT (total AT), SCAT, and abdominal AT (AAT) volumes were evaluated at $\mathrm{T} 12$ as described previously [24]. Briefly, isoflurane-anesthetized rats were laid in an imaging cell (Minerve, Esternay, France) and threedimensional X-ray images were acquired on a $\mu$ SPECTmicro-computed tomography (eXplore speCZT Vision 120, GE, Waukesha, USA). All volumes were reconstructed with a voxel size equal to $200 \times 200 \times 200 \mu \mathrm{m}^{3}$. Reconstructed images were filtered with a Gaussian filter to reduce noise. One control rat was used to determine thresholds, based on the Hounsfield unit (HU), to isolate fat tissues from the other structures; threshold values obtained (range from -390 to $-200 \mathrm{HU}$ ) were then applied to all other rats. The proportion of fat was calculated as the ratio between the volume of AT and the whole body volume.

\section{Glucose tolerance tests and insulin sensitivity}

Glucose tolerance after intraperitoneal (i.p.) injection was measured at $\mathrm{T} 0, \mathrm{~T} 4, \mathrm{~T} 8$, and $\mathrm{T} 12$ in conscious rats; glucose $(2 \mathrm{~g} / \mathrm{kg}$ ) was administered at $t=0$ and plasma glucose was determined after $15,30,60$, and $120 \mathrm{~min}$. At $\mathrm{T} 12$, rats anesthetized with pentobarbital $(50-60 \mathrm{mg} / \mathrm{kg}$, i.p.) were randomized to undergo either a glucose tolerance test after i.v. injection (IVGTT) or an insulin sensitivity test. For IVGTT, glucose $(0.5 \mathrm{~g} / \mathrm{kg})$ was administered at $t=0$; plasma glucose and insulin concentrations were then determined after $3,6,10,15,30,45$, and $60 \mathrm{~min}$, and expressed as percentages of values measured at $t=0$. To test insulin response and sensitivity, insulin $(1.5 \mathrm{U} / \mathrm{kg})$ was administered i.v. at $t=0$; plasma glucose was determined after 10, 15, 30, 45, and $60 \mathrm{~min}$, and expressed as percentage of values measured at $t=0$. In all experiments, the area under the curve (AUC) was calculated from the glucose and/or insulin values. Biopsies of liver were sampled 10 and 60 min following insulin administration, to evaluate the activation of the insulin transduction pathways (AKT) by western blotting (see below); biopsies of ATs were also sampled at $t=60 \mathrm{~min}$ to evaluate the membrane expression of the glucose transporter Glut4 by immunostaining (see below).

\section{AT histology and immunostaining}

Fixed AT was embedded in paraffin. Eight-micrometer slices were cut and stained with hematoxylin/eosin. Microscopic images were captured using a video camera coupled to a computer (Leica Microsystems, Heerbrugg, Switzerland) and analyzed with the Image $\mathbf{J}$ software to measure the size of adipocytes $\left(\mu \mathrm{m}^{2}\right)$. For each animal, given values represent the mean of 50 representative adipocytes from five randomly chosen images. For immunostaining, $4 \mu \mathrm{m}$ slices were cut and incubated with anti-Glut4 or anti-Ibal protein primary antibodies and appropriate secondary antibodies (Alexa Fluor 555 and biotinconjugated anti-rabbit IgG, respectively). Immunostaining was analyzed with Image $\mathbf{J}$ software on six microscopic images randomly chosen from three to five slices and expressed as the mean \% area of specific labeling over the total area. Control slices without primary antibodies were used to assess nonspecific labeling. 


\section{Studies in human-derived hepatocarcinoma HepG2 cells}

Human-derived hepatocarcinoma HepG2 cells (kindly obtained from F. Pons, CAMB - UMR7199, Faculté de Pharmacie, Illkirch, France) were cultured in $75 \mathrm{~cm}^{2}$ flasks in Dulbecco's modified Eagle's medium (DMEM) F12 without phenol red containing $10 \%$ heat-inactivated fetal calf serum and $2 \%$ penicillin/streptomycin. Cells were incubated in a humidified atmosphere of $5 \% \mathrm{CO}_{2}$ at $37^{\circ} \mathrm{C}$ and passaged every 3 days by trypsinization (trypsinEDTA). For experiments, HepG2 cells were incubated in 6or 24-well plates in complete medium, grown to $70-80 \%$ confluence, then maintained in serum-free DMEM for $5 \mathrm{~h}$ before treatments. Under these conditions, LNPs up to $10^{-3}$ M displayed no cell toxicity (data not shown).

AKT, AMPK $\alpha$, and acetyl-CoA carboxylase (ACC) phosphorylations were triggered by exposing cells to insulin for $10 \mathrm{~min}$ and/or to LNP509, LNP599, and AICAR (cellpermeable AMP analog acting as AMPK activator) for $60 \mathrm{~min}$. In some experiments, cells were pre-treated with the $\mathrm{I}_{1} \mathrm{R}$ antagonist, efaroxan, or the AMPK inhibitor, compound C (6-[4-(2-piperidin-1-ylethoxy) phenyl]-3-pyridin-4ylpyrazolo [1,5-a]pyrimidine) for 30 and $90 \mathrm{~min}$, respectively. The AMPK $\alpha$ phosphorylated on Thr172 (pAMPK $\alpha) /$ AMPK $\alpha$, AKT phosphorylated on Ser473 (pAKT)/AKT, and ACC phosphorylated on Ser79 (pACC)/ACC ratios were calculated by western blotting as described below. Intracellular lipid accumulation (in vitro model of steatosis) was induced by exposing HepG2 cells to oleic acid ( $2 \mathrm{mM})$ in the presence or absence of LNP509 or LNP599. After $24 \mathrm{~h}$, the medium was removed, cells were washed twice with phosphate-buffered saline solution and fixed with formalin $(10 \%)$ at room temperature for $1 \mathrm{~h}$. Control cells were treated with oleic acid-free medium. Intracellular lipid deposits were stained with Oil Red O (60\%) and examined under light microscopy. Quantification of cell lipid content was achieved spectrophotometrically by measuring optic density at $492 \mathrm{~nm}$ after elution of Oil Red O staining with isopropanol $60 \%$.

\section{Immunoblotting}

Tissues or HepG2 cells were homogenized in HEPES buffer containing protease and phosphatase inhibitors (Complet EDTA and PhosStop, Roche Diagnostics), then centrifuged for $10 \mathrm{~min}$ at $10,000 \times g$ at $4{ }^{\circ} \mathrm{C}$. Total AMPK $\alpha$, pAMPK $\alpha$, total AKT, pAKT, total ACC, pACC, total NFkB p65, and NFkB p65 phosphorylated on Ser536 (pNFkB) were probed using appropriate rabbit antibodies purchased from Cell Signaling; the antibody probing uncoupling protein-1 (UCP-1) was from Abcam; all antibodies were incubated overnight at $4{ }^{\circ} \mathrm{C}$ with gentle shaking. Reactive proteins were detected and quantified using the BioRad Chemidoc System and Image Lab (BioRad) software. AMPK $\alpha$, AKT, and NFkB p65 activations, and ACC inhibition were eval-

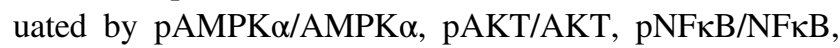
and $\mathrm{pACC} / \mathrm{ACC}$ ratios, respectively. In tissue lysates, data were expressed relative to the mean value of the control group. In HepG2 cell homogenates, data were normalized with non-stimulated or AICAR-stimulated cells.

\section{Statistics}

Results are presented as means \pm SEM of $n=11$ controls and $n=12$ LNP599-treated rats, unless otherwise stated. Treatments were compared using one-way analysis of variance (ANOVA) followed by a Bonferonni's post-hoc test or unpaired two-sample $T$-test, as specified in Figure legends. Comparisons within one group (age-dependent changes) were performed using paired two-sample $T$-test. Percentages of changes were calculated as (value at T12 value at T0) $\times 100 /$ value at T0; comparisons were performed using a one-sample $T$-test with the $\%$ change in controls used as reference value. In HepG2 cell experiments, data are expressed as means \pm SEM of $n$ independent experiments. Differences were analyzed by unpaired twosample $T$-test.

$P$-values $<0.05$ were considered as statistically significant.

\section{Results}

\section{Effects of LNP599 treatment on body weight, fat mass, food intake, and body temperature}

Body weight increased significantly less in rats treated with LNP599 compared with controls so that the total weight gain was $32 \%$ lower after 12 weeks of treatment (Fig. 1a). This was associated with a significant reduction in the total AT estimated by $\mu \mathrm{CT}$, resulting mainly from a (nonsignificant) decrease in SCAT $(P=0.0597)$ with no change in AAT (i.e., visceral and epididymal) (Fig. 1b). This was confirmed at autopsia: the mass of SCAT was decreased in treated animals, whereas that of EAT was unchanged (Fig. 1c); both were significantly correlated with the boby weight (Fig. 1d). The mass of interscapular (brown) AT was similar $(9.30 \pm 1.87 \mathrm{~g} / \mathrm{kg}$ and $11.05 \pm 1.70 \mathrm{~g} / \mathrm{kg}$ in LNP599 and control groups, respectively; $P=0.2470$ ). Reduced weight gain in LNP599-treated rats was not related to a change in $24 \mathrm{~h}$ food intake (Fig. 1e left); however, the proportion of food consumption during the night period (i.e., from 19:00 to 07:00 h) was increased in these animals at T12 compared with $\mathrm{T} 0(69 \pm 2 \%$ and $62 \pm$ $1 \%$, respectively; $P=0.0096$ ) and compared with untreated 

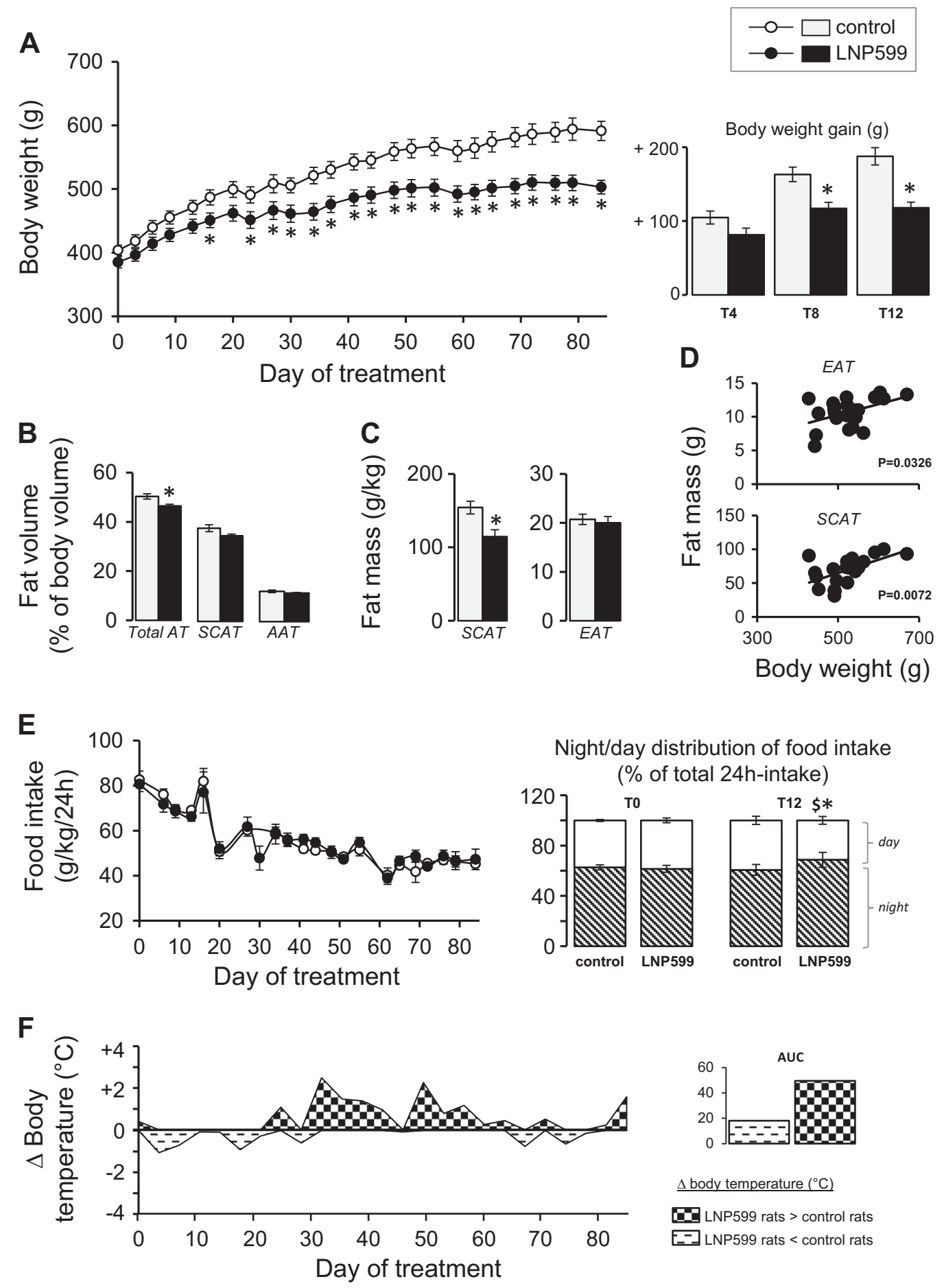

$\underline{\Delta \text { body temperature }\left({ }^{\circ} \mathrm{C}\right)}$

[플 LNP99 rats > control rats

E- LNP599 rats < control rats

Fig. 1 Effects of LNP599 treatment on body weight, fat mass, food intake, and body temperature. LNP599 reduced a body weight gain and total and subcutaneous adiposity estimated by $\mathbf{b} \mu \mathrm{CT}$ and $\mathbf{c}$ postmortem fat pad mass. d Epididymal and subcutaneous fat mass were significantly correlated with body weight. e Total $24 \mathrm{~h}$ food intake was

animals $(61 \pm 1 \%$ at T12; $P=0.0015)$ (Fig. 1e right). From the third week of treatment onwards, body temperature measured at the interscapular level was almost always unchanged but night/day distribution of food intake was increased. f From the third week of treatment, body temperature was almost constantly higher in treated rats compared with controls. $* P<0.05$ : LNP599 vs. control rats at the same age (one-way ANOVA). $\$ P<$ 0.05: T12 vs. T0 within the same group (paired $T$-test)

higher in treated animals than in controls (mean temperature $=35.1 \pm 0.1{ }^{\circ} \mathrm{C}$ and $34.7 \pm 0.1^{\circ} \mathrm{C}$, respectively; $P=$ 0.0153) (Fig. 1f). 

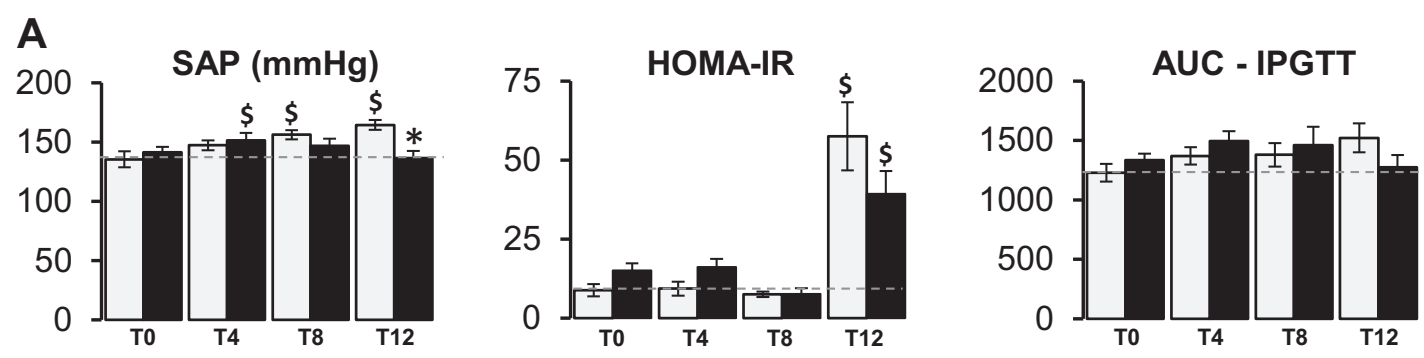

Total Cs (mmol/L)
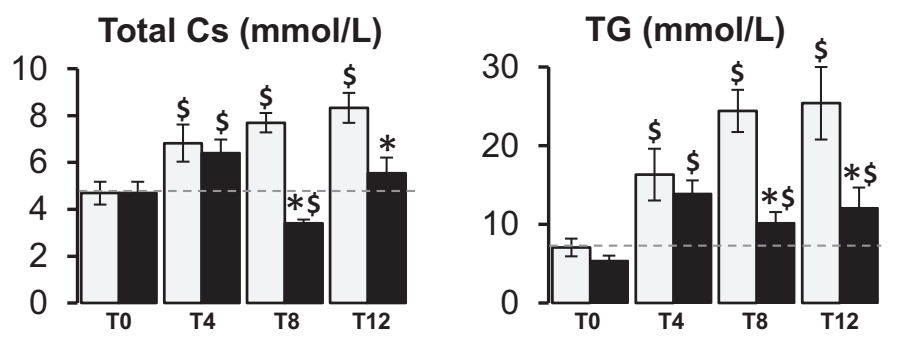

B

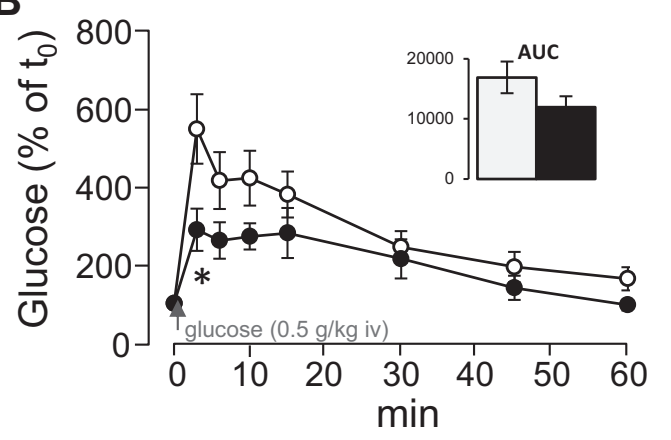

C
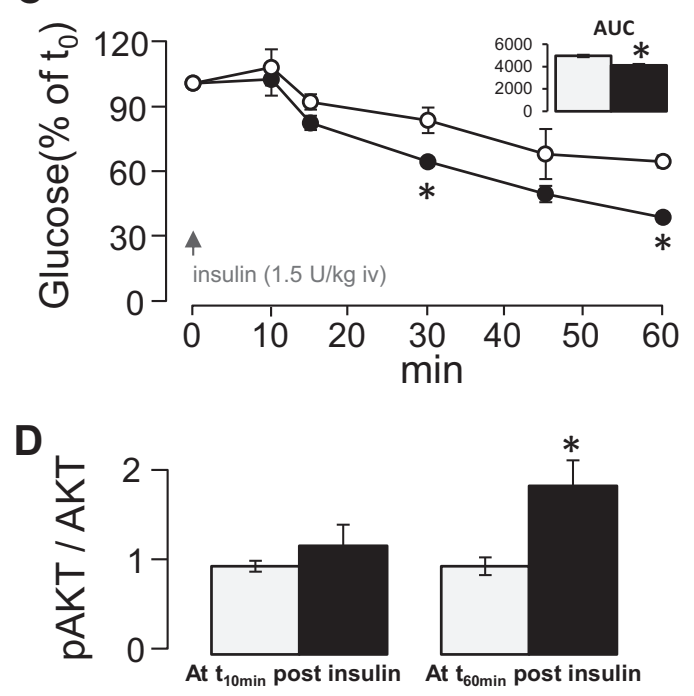

\section{LNP599 treatment improves cardiovascular and metabolic homeostasis}

LNP599 reduced the age-dependent increase in systolic blood pressure, total cholesterol, and TGs in plasma, glucose intolerance, and the HOMA-IR (Fig. 2a). At the end of

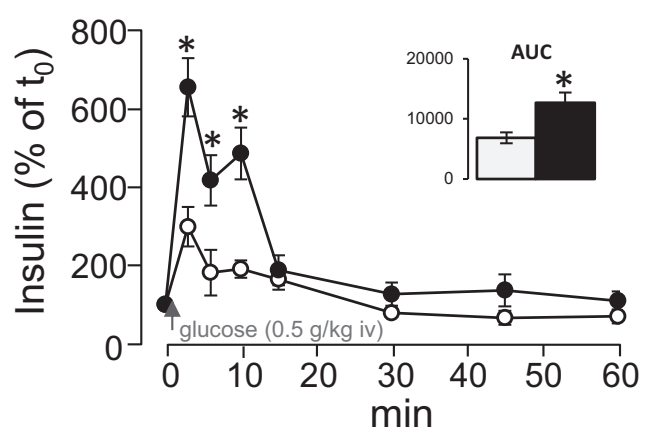

E
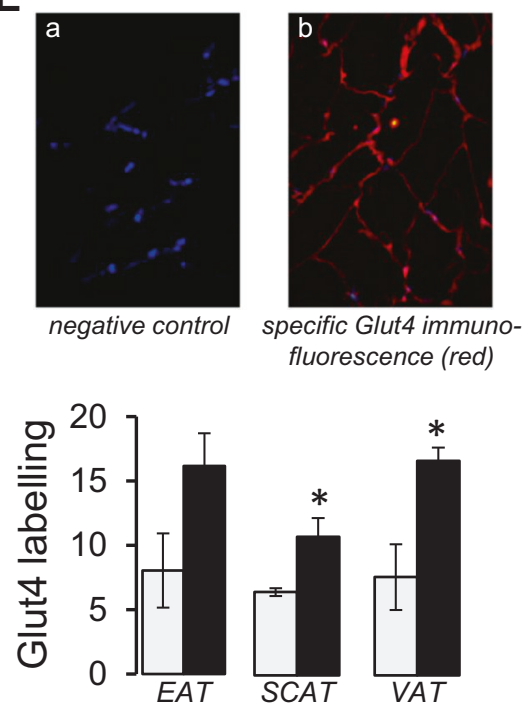

the treatment period, most parameters were improved in treated animals compared with controls (Table 1 and Fig. 2a). After i.v. glucose challenge (Fig. 2b left), the peak glycemia was almost twice lower in LNP599-treated rats $(n=9)$ compared with controls $(n=8)$; in parallel, plasma insulin increased markedly more and the calculated AUC 
Fig. 2 Twelve-weeks LNP599 treatment limits the development of cardiovascular and metabolic dysfunctions. a From T0 to T12, untreated Zucker rats developed hypertension (indicated by an increased systolic arterial pressure, SAP), impaired carbohydrate regulation (glucose intolerance assessed by the AUC following glucose challenge at $2 \mathrm{~g} / \mathrm{kg}$ i.p. and insulin resistance evaluated by the HOMAIR index) and severe dyslipidemia (plasma total cholesterol and triglycerides). LNP599 limited or even prevented these age-dependent alterations. At T12, glucose tolerance and insulin sensitivity were significantly improved in LNP599-treated rats compared with controls. b) After challenging with glucose, glycemia increased less, whereas glucose-stimulated insulin secretion (GSIS) was higher. c Hypoglycemic effect of insulin administration was enhanced, as well as d AKT phosphorylation in hepatic tissues and e glucose transporter Glut4 membrane expression in epididymal (EAT), subcutaneous (SCAT), and visceral (VAT) adipose tissues $[\mathrm{a}, \mathrm{b}$ : representative images of negative control displaying nuclei stained with Hoechst but no Glut4 labeling (a) and specific Glut4 immunolabelling (b)]. $* P<0.05$ : LNP599 vs. control rats (one-way ANOVA $(\mathbf{a}, \mathbf{b})$ or unpaired $T$-test (c-e)). $\$ P<0.05$ : T4, T8, or T12 vs. T0 within the same group (paired $T$-test)

nearly doubled (Fig. 2b right). Insulin sensitivity was improved in animals receiving LNP599 compared with control rats ( $n=3$ in each group), as demonstrated by the potentiated hypoglycemic effects of insulin administration (Fig. 2c) and increased AKT phosphorylation (Fig. 2d) and glucose transporter Glut4 membrane expression (Fig. 2e) in insulin-sensitive tissues.

\section{LNP599 treatment protects adipose and hepatic tissues}

In SCAT and EAT from control rats, the size of adipocytes progressively increased over weeks (Fig. 3a). LNP599 tempered these age-related morphological changes, so that, at $\mathrm{T} 12$, the size of adipocytes was significantly different between the two groups (see in Fig. 3a representative histological sections of controls [a, c] and LNP599-treated rats $[b, d])$. Low-grade inflammation, quantified at the end of the treatment by macrophage infiltration and activation of the transcription factor NFKB, was reduced by $34-56 \%$ (Fig. 3b, c; $n=3$ in each group). Regarding the visceral AT sampled at T12 (Fig. 3a [e, f], 3b, 3c), similar differences between controls and LNP599 rats were evidenced. Interscapular AT sampled at T12 also displayed morphological remodeling (Fig. 3a [g, h]) and significantly higher ( $\times 2.5$ vs. controls, $P=0.0117$ ) UCP-1 expression (Fig. 3d). Plasma adiponectin levels were $30 \%$ higher in treated rats compared with controls (Table 1).

LNP599 treatment markedly stimulated AMPK $(+57 \%$ in the pAMPK $\alpha / \mathrm{AMPK} \alpha$ ratio; $P=0.1370$ vs. controls) and significantly enhanced the inhibitory phosphorylation of ACC, indicating reduced enzyme activity (Fig. 4a). Blood markers of hepatic damage or disease (aspartatealanine aminotransferases and alkaline phosphatase) were decreased by $30-40 \%$ (Fig. 4b), as were pro-inflammatory cytokine levels (Fig. 4c).

\section{LNP599 improves cell metabolism and prevents steatosis in hepatocytes}

Direct effects of LNP599 (and the reference ligand for in vitro experiments, LNP509 [15]) on liver regulatory pathways of glucose and lipid homeostasis were studied in HepG2 cells.

LNPs alone did not change the pAKT/AKT ratio but significantly potentiated insulin-induced AKT activation $(\mathrm{pAKT} / \mathrm{AKT}=4.38 \pm 0.63$ for LNP509 and $4.40 \pm 0.68$ for LNP599; $P=0.0068$ and 0.0081 , respectively, vs. $1.97 \pm$ 0.29 in insulin-stimulated cells); these potentiating effects
Table 1 Cardiovascular and metabolic parameters before (T0) and after (T12) 12-weeks treatment with LNP599 $(10 \mathrm{mg} / \mathrm{kg} / \mathrm{d})$

\begin{tabular}{lcccc}
\hline & Baseline (T0) & & \multicolumn{2}{l}{ End of treatment (T12) } \\
& Control & LNP599 & Control & LNP599 \\
\hline Body weight $(\mathrm{g})$ & $404 \pm 8$ & $383 \pm 9$ & $598 \pm 16(+48 \%)$ & $510 \pm 12(+33 \%)^{\mathrm{a}}$ \\
Norepinephrine (pmol/L) & $1198 \pm 182$ & $1127 \pm 163$ & $1021 \pm 448(+1 \%)$ & $472 \pm 145(-46 \%)^{\mathrm{a}}$ \\
Systolic blood pressure & $135 \pm 7$ & $141 \pm 5$ & $164 \pm 4(+23 \%)$ & $136 \pm 6(-3 \%)^{\mathrm{a}}$ \\
$(\mathrm{mm}$ Hg) & & & & \\
Glucose tolerance (AUC- & $1229 \pm 74$ & $1335 \pm 54$ & $1522 \pm 123(+24 \%)$ & $1276 \pm 103(-2 \%)^{\mathrm{a}}$ \\
IPGTT) & & & & \\
Fasting glucose (mmol/L) & $5.9 \pm 0.3$ & $6.4 \pm 0.2$ & $6.4 \pm 0.4(+10 \%)$ & $7.3 \pm 0.5(+14 \%)$ \\
Fasting insulin ( $\mu \mathrm{g} / \mathrm{L})$ & $1.51 \pm 0.38$ & $2.30 \pm 0.39$ & $8.47 \pm 1.66(+720 \%)$ & $5.54 \pm 1.25(+115 \%)^{\mathrm{a}}$ \\
HOMA-IR & $8.9 \pm 1.9$ & $15.1 \pm 2.3$ & $57.5 \pm 10.8(+869 \%)$ & $39.3 \pm 7.2(+131 \%)^{\mathrm{a}}$ \\
Adiponectin $(\mathrm{mg} / \mathrm{L})$ & $2.27 \pm 0.22$ & $2.55 \pm 0.28$ & $2.36 \pm 0.35(-2 \%)$ & $3.08 \pm 0.67(+26 \%)$ \\
Total cholesterol $(\mathrm{mmol} / \mathrm{L})$ & $4.7 \pm 0.5$ & $4.7 \pm 0.5$ & $8.3 \pm 0.6(+93 \%)$ & $5.5 \pm 0.7(+14 \%)^{\mathrm{a}}$ \\
Triglycerides $(\mathrm{mmol} / \mathrm{L})$ & $7.1 \pm 1.1$ & $5.4 \pm 0.6$ & $25.4 \pm 4.6(+301 \%)$ & $12.1 \pm 2.6(+174 \%)^{\mathrm{a}}$ \\
\hline
\end{tabular}

${ }^{\text {a }} P<0.05$ : LNP599 vs. control rats (one-sample $T$-test).

Values in brackets indicate the mean \% change between T0 and T12 
Fig. 3 Twelve-weeks LNP599 treatment protects adipose tissue morphology and reduces local inflammation. a The mean size of adipocytes increased from T0 to T12 in control animals; these age-dependent changes were significantly less pronounced in animals receiving LNP599 treatment [representative histological sections from control (a, c, e, g) and LNP599. treated $(b, d, f, h)$ rats at $\mathrm{T} 12]$. Inflammatory markers (macrophage infiltration of adipose tissues assessed by Iba1 protein labeling (b) and activation of NFkB in tissue lysates (c)) evaluated at T12 were lowered by LNP599 treatment [arrows indicate Iba1 protein immunolabeling in representative histological sections]. d Expression of UCP1 in the interscapular adipose tissue of treated rats was more than twice that of control rats. $* P<0.05$ : LNP599 vs. control rats (one-way ANOVA). $\$ P<$ 0.05: T8 or T12 vs. T0 within the same group (paired $T$-test)
A
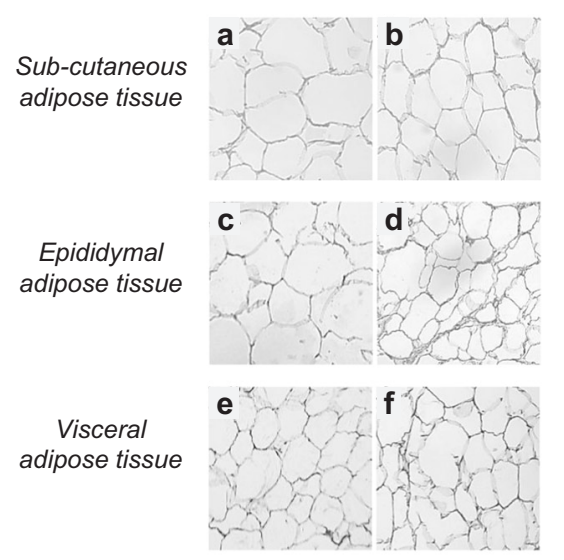

Interscapular adipose tissue

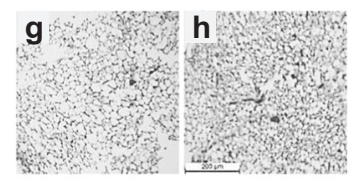

B
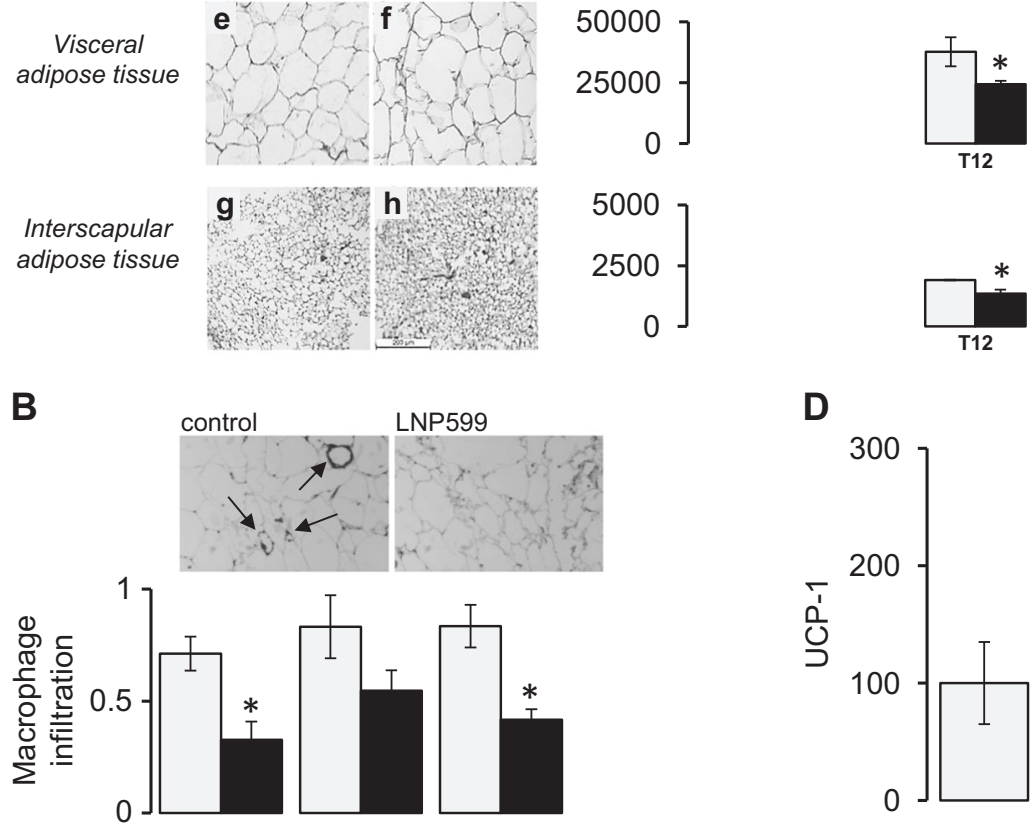

$\left.\begin{array}{r}5000 \\ 2500 \\ 0\end{array}\right]$

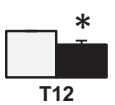

D

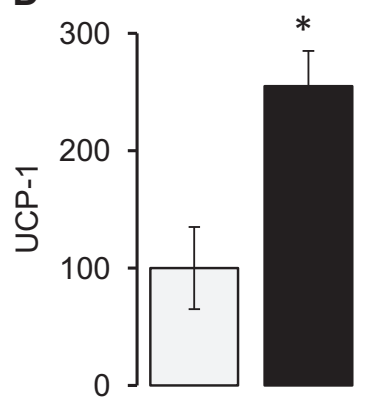

C

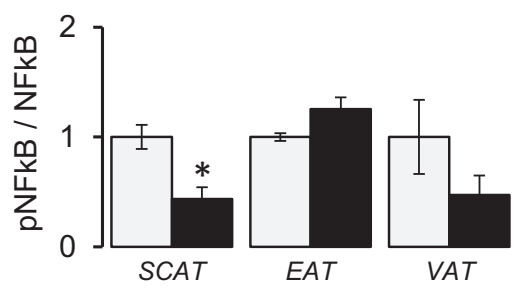

were abolished by efaroxan (Fig. 5a; $n=3$ ). LNPs triggered AMPK $\alpha$ phosphorylation similar to the direct AMPK $\alpha$ activator AICAR (Fig. 5b; $n=4-6$ ). In another series of experiments $(n=4)$, pretreatment with compound $\mathrm{C}$ abolished AMPK $\alpha$ activation by LNP509 (Fig. 5c right panel) without affecting the $\approx 2$-fold increase in the pAKT/AKT ratio in cells exposed to LNP509 + insulin compared with cells exposed to insulin alone (Fig. 5c left panel), thus excluding that the potentiating action of LNPs on insulin signaling is downstream of AMPK $\alpha$ activation.

Activity of ACC, one of the substrate of AMPK $\alpha$, was markedly suppressed by LNPs, as shown by the 2.5- to 3fold increase in pACC/ACC ratio (Fig. $5 \mathrm{~d} ; n=5$ ). Finally,
LNPs tended to prevent the intracellular lipid accumulation in cells exposed to oleic acid (Fig. 5e; $n=4$ ).

\section{Discussion}

The present results confirm the beneficial effects of LNP599 on most of the cardiovascular and metabolic parameters defining the MetS. In addition, they demonstrate for the first time (i) that LNP599 limits the age-dependent expansion and inflammation of the white ATs and favors the thermogenic function of BAT and energy expenditure, (ii) that these effects result in reduced total fat deposits and 

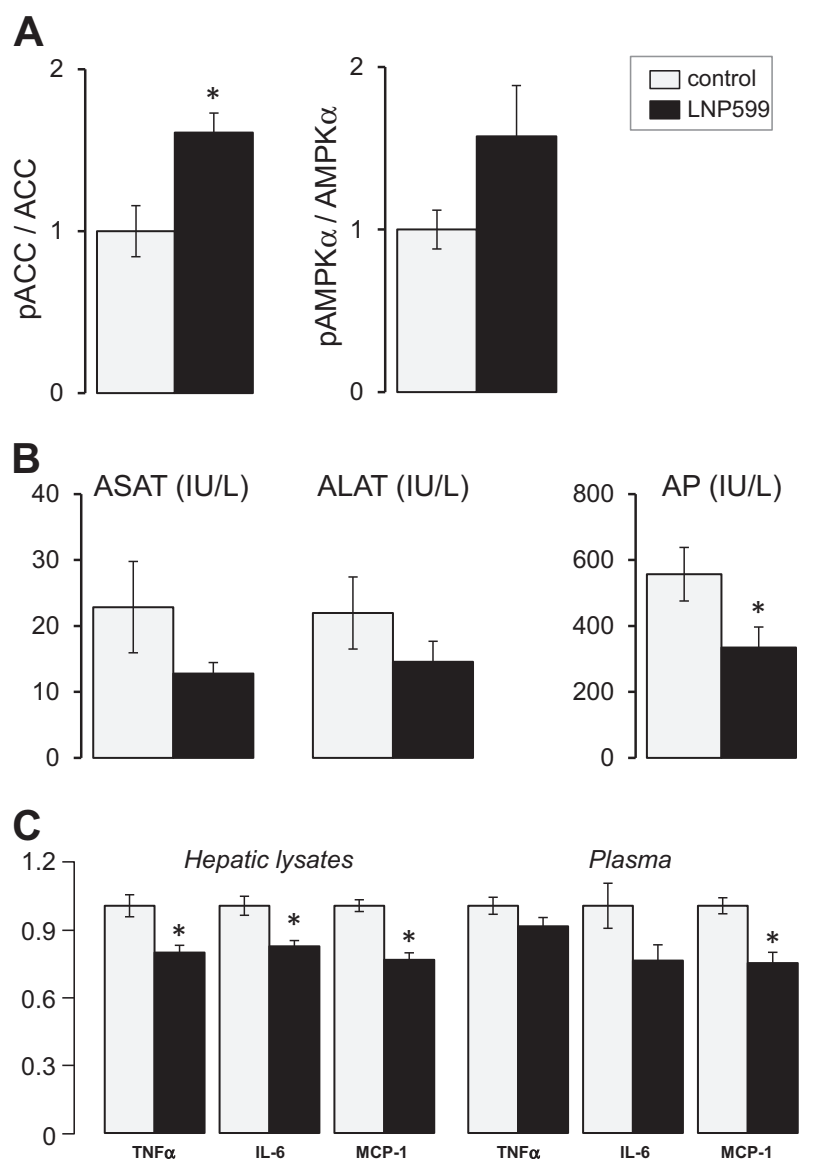

Fig. 4 Twelve-weeks LNP599 treatment protects hepatic function. Phosphorylation of AMPK $\alpha$ and ACC, two key enzymes of hepatocyte metabolism, was increased in hepatic lysates of rats receiving LNP599 (a). Plasma levels of aspartate aminotransferase (ASAT), alanine aminotransferase (ALAT), and alkaline phosphatase (AP) were decreased (b). Inflammatory cytokine levels were reduced in hepatic lysates and in plasma $(\mathbf{c}) . * P<0.05$ : LNP599 vs. control rats (one-way ANOVA)

attenuated body weight gain, and finally, (iii) that the improvement of the metabolic profile by LNP599 is to be linked mostly to its protective action against obesity development. Accordingly, the kinetic of occurrence of these effects was similar. Compared with age-matched Sprague-Dawley (present data) and lean Zucker rats [25, 26], fa/fa Zucker rats at baseline were normotensive and normoglycemic, but had higher insulin, cholesterol, and TG levels. Over the 12 weeks of the study, untreated animals developed hypertension and glucose intolerance, and the severity of insulin resistance and dyslipidemia increased dramatically. In contrast, all these age-dependent functional alterations were markedly attenuated from the fourth to eighth week of LNP599 treatment. In parallel, the body weight tended to stabilize and less AT remodeling occurred. Also, most of the cardiovascular and metabolic parameters measured at the end of the treatment period were correlated with the body weight (Supplementary Fig. 2A) and various indices of adiposity (not shown) and changes in these parameters were strongly related to the weight gain over the 12-weeks period (Supplementary Fig. 2B). These data are in full agreement with several studies in humans showing that decreases in body weight and/or in fat mass significantly improve insulin resistance and glucose and lipid homeostasis, and reduce arterial blood pressure [27-29]. Of note, and in contrast to our previous observation using LNP509 [15], the glucose-stimulated insulin secretion (GSIS) was increased by LNP599. This effect was independent from changes in pancreas morphology, as the number and the size of Langerhans islets were unchanged by LNP599 (not shown); it was most probably also not due to any direct effect on $\beta$-pancreatic cells, as the glucose-induced insulin release by RINm5F pancreatic $\beta$-cells was not modified by LNP599 (not shown). Increased GSIS in the present study could be related to the additional central sympathoinhibitory action of LNP599 compared with LNP509. It is established that obesity is associated with sympathetic hyperactivity, autonomic dysfunction, and blunted sympathetic responses to an oral glucose challenge in humans and Zucker rats [30-32]. Thus, reducing the sympathetic tone with LNP599 could improve autonomic control of pancreatic $\alpha$ - and $\beta$-cell responses to acute hyperglycemia. However, GSIS was not correlated to norepinephrine plasma levels or the degree of sympatho-inhibition, arguing against this hypothesis. Alternatively, reduced obesity in rats receiving LNP599 may have reversed the pancreatic cell responsiveness, as demonstrated by Straznicky et al. [29] in obese insulin-resistant subjects. Accordingly, the body weight at the end of the treatment period was inversely correlated to GSIS (Supplementary Fig. 2A).

Reduction of body weight gain and prevention of fat accumulation upon LNP599 treatment was not due to caloric restriction, as food intake was similar in all animals. It may then rather be related to enhanced energy expenditure. In agreement, the behavior of feeding was shifted to more intense feeding during the night, i.e., during murineactive period. Moreover, the body temperature was slightly but constantly increased and expression of UCP-1 in interscapular (brown) AT lysates was more than doubled, suggesting potentiated thermogenesis. In the last decade, dysfunction of BAT has been extensively described as one of the cause of development of obesity in human [22, 33, 34]. In constrast, Peterson et al. [35] found no correlation between weight gain and BAT thermogenic activity in overfed subjects, questioning the role of this particular AT in the prevention of obesity. However, in the latter study, overfeeding was conducted in a short period of time, induced only moderate body weight increases (5-15\%), and no change in cardio-metabolic parameters. From our data, one may assume that chronic administration of LNP599 
Fig. 5 LNPs improve hepatocyte metabolism and prevent intracellular lipid accumulation in cultured human HepG2 cells. a Pretreatment with LNP599 or LNP509 potentiated AKT activation induced by insulin and these effects were abolished by the $\mathrm{I}_{1} \mathrm{R}$ antagonist, efaroxan. Both induced AMPK $\alpha$ phosphorylation similar to the AMPK $\alpha$ direct activator AICAR (b); blockade of the LNPinduced AMPK $\alpha$ phosphorylation by compound $\mathrm{C}$ (c, right panel) did not affect its stimulatory action on AKT signaling (c, left panel). LNPs induced significant phosphorylation of ACC (d) and tended to prevent intracellular lipid accumulation in HepG2 cells exposed to oleic acid (e) [arrows indicate lipid droplets stained with Oil Red O in representative cultured HepG2 cells]. $* P<0.05$ vs. insulinstimulated cells (unpaired $T$ test). $£ P<0.05$ vs. nonstimulated cells (unpaired $T$-test)
A

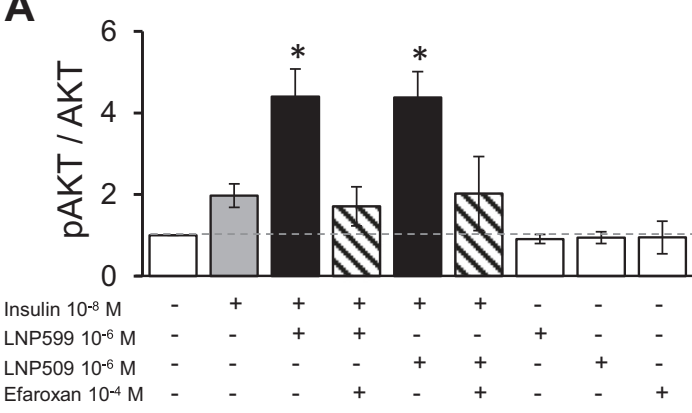

C
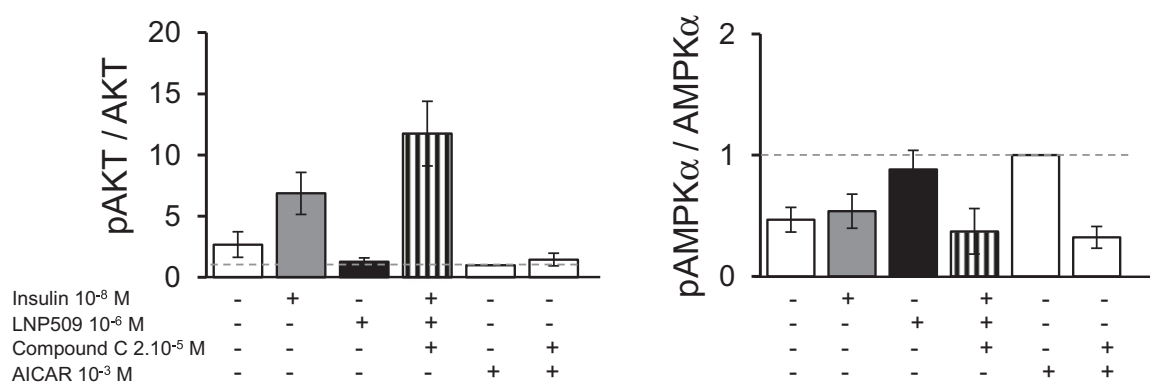

D

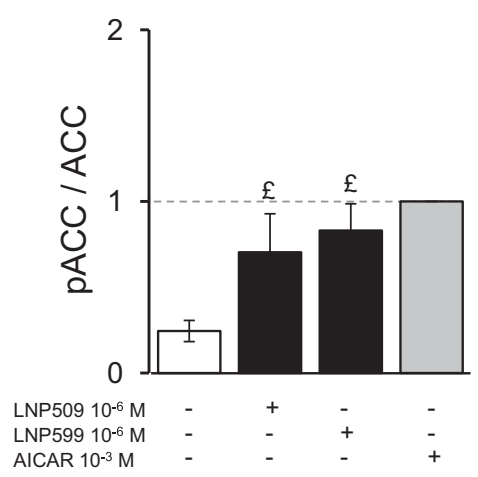

B

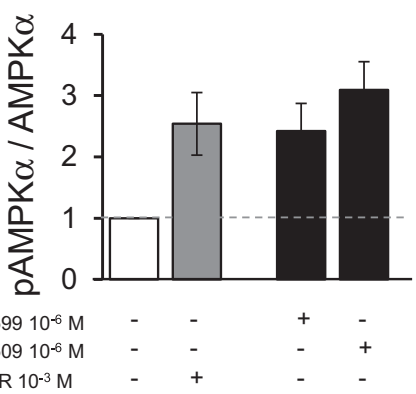

E
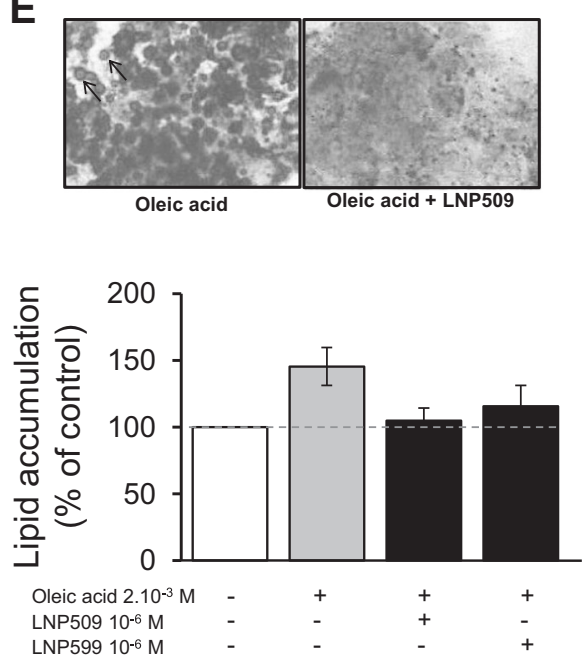

restored-or at least improved-BAT functions and thus maintained energy balance in the treated rats.

Neither body weight nor cardiovascular and metabolic parameters were significantly correlated to plasma norepinephrine levels or to the degree of sympatho-inhibition, even when regression analysis were performed including only the treated subgroup. These observations suggest that the LNP599-induced prevention of obesity in this experimental model relies mostly on additional, i.e., independent from sympatho-inhibition, effect(s).

We previously demonstrated that stimulation of $I_{1} R$ on white adipocytes increases adiponectin secretion, likely through activation of peroxisome proliferator-activated receptor- $\gamma(\operatorname{PPAR} \gamma)$ [15]. In line with these early results, plasma adiponectin was $\approx 30 \%$ higher in animals receiving
LNP599 in the present study; this increase is of the same range as those previously reported by us and others in Zucker rats [36] and may be clinically relevant. The multiple beneficial actions of adiponectin on inflammation, cardio-metabolic homeostasis, obesity, and adiposity have been extensively described. Regarding BAT, adiponectin has been shown to attenuate weight gain and fat-pad deposition in a mouse model of obesity by activating BAT and increasing energy expenditure, in the absence of change in food intake [37]. Direct effects of LNP599 on brown adipocytes, as described on white adipocytes, could also occur. Interestingly, PPAR $\gamma$ activation stabilizes and potentiates the activity of the transcriptional regulator PRDM16, an essential determinant of BAT differentiation and function [38, 39]. Such LNP599-induced PPAR $\gamma$ 
activation in brown adipocytes could explain the enhanced UCP-1 levels observed in our animals (see above). Further studies are needed to fully characterize this new effect of $I_{1}$ ligands in BAT.

Another major new finding of the present study is that LNPs can also act directly on liver $\mathrm{I}_{1} \mathrm{R}$ to improve both carbohydrate and lipid metabolisms. Incubation of cultured hepatocytes with LNPs activates AMPK, resulting in phosphorylation (i.e., inactivation) of ACC and thus in reduced lipogenesis and amplified fatty acid oxidation. Similar effects on AMPK and ACC activities are found in hepatic lysates of treated Zucker rats, together with several evidences for a global improvement of hepatic function (reduced levels of blood markers of hepatic disease and inflammatory cytokines). It was beyond the scope of the present study to clarify the molecular mechanisms involved in the LNP-induced AMPK activation. Still, several hypothesis can be proposed: (i) increase of the AMP:ATP ratio through inhibition of Complex I of the mitochondrial respiratory chain (similarly to the antidiabetic metformin), (ii) action as a pro-drug converted into an AMP analog (similar to the reference AMPK activator, AICAR, and C13), and finally (iii) activation of signaling pathways leading to increases in cytosolic $\mathrm{Ca}^{++}$and consequently to activation of the $\mathrm{Ca}^{++} /$calmodulin-dependent kinase CaMKK $\beta$ and AMPK phosphorylation. As AMPK activation by LNPs is sensitive to $\mathrm{I}_{1} \mathrm{R}$ blockade [15] and as several $\mathrm{I}_{1}$ agonists have been shown to activate $\mathrm{PKC}$ and $\mathrm{Ca}^{++}$ influx in cells [40-42], the latter hypothesis may be favored. LNPs also potentiate insulin action, as shown by the two fold increase in the pAKT/AKT ratio in insulin-stimulated cultured hepatocytes. This effect is independent from AMPK activation, as it persists in the absence of AMPK phosphorylation. Potentiation of insulin signaling by LNPs may involve actions at insulin-receptor substrate 1 (IRS-1); in agreement, moxonidine, a non-selective $\mathrm{I}_{1} \mathrm{R}$ agonist, has been shown to enhance both expression and insulin-induced phosphorylation of IRS-1 in the liver and skeletal muscle [43]. Allosteric positive modulation of the insulin receptor or reduced phosphatase and tensin homolog-mediated dephosphorylation of the upstream phosphatidylinositol 3kinase by LNPs can also not be ruled out. All together, LNPs's direct effects on hepatocytes may temper the development of obesity and adiposity in treated animals, both directly through AMPK-mediated inhibition of lipogenic gene expression and indirectly through improvement of insulin action and glucose and lipid profile, and reduction of lipotoxicity [20, 44].

In conclusion, the present data show that activation of $\mathrm{I}_{1}$ receptors on hepatocytes stimulates the cellular energy sensor AMPK and potentiates insulin signaling. Furthermore, $I_{1}$ ligands favor the thermogenic function of BAT and energy expenditure; whether a direct action at brown adipocytes is involved remains to be elucidated. Stimulation of the AMPK pathways and insulin sensitization in the liver and possibly other insulin-sensitive tissues (e.g., skeletal muscles) can also be achieved indirectly through the $\mathrm{I}_{1^{-}}$ mediated enhanced adiponectin secretion by white adipocytes, as demonstrated previously. All these cell effects may act synergistically to prevent lipogenesis and obesity development, and interrupt the vicious circle relating obesity, inflammation, and insulin resistance, thus leading to improved glucose and lipid homeostasis. Selective $\mathrm{I}_{1} \mathrm{R}$ ligands could therefore prove beneficial as novel protective drugs against the development of adiposity and obesity, and associated cardio-metabolic disorders.

Acknowledgements This work was financially supported by the SATT Conectus Alsace, the University of Strasbourg, and the Région Alsace (fellowship to MW). We acknowledge Stéphanie Dal and Elodie Seyfritz from the Centre Européen d'Etude du Diabète for their advice and help at imunofluorescence analysis, and Lucie Tischmacher for her technical assistance.

\section{Compliance with ethical standards}

Conflict of interest The authors declare that they have no conflict of interest.

Publisher's note: Springer Nature remains neutral with regard to jurisdictional claims in published maps and institutional affiliations.

\section{References}

1. Alberti KG, Zimmet P, Shaw J. Metabolic syndrome-a new world-wide definition. A consensus statement from the International Diabetes Federation. Diabet Med. 2006;23:469-80.

2. Grundy SM, Cleeman JI, Daniels SR, Donato KA, Eckel RH, Franklin BA, et al. Diagnosis and management of the metabolic syndrome: an American Heart Association/National Heart, Lung, and Blood Institute Scientific Statement. Circulation. 2005;112: 2735-52.

3. Harris MF. The metabolic syndrome. Aust Fam Physician. 2013; 42:524-7.

4. Grassi G, Dell'Oro R, Quarti-Trevano F, Scopelliti F, Seravalle G, Paleari F, et al. Neuroadrenergic and reflex abnormalities in patients with metabolic syndrome. Diabetologia. 2005;48:1359-65.

5. Mancia G, Bousquet P, Elghozi JL, Esler M, Grassi G, Julius S, et al. The sympathetic nervous system and the metabolic syndrome. J Hypertens. 2007;25:909-20.

6. Feldstein C, Julius S. The complex interaction between overweight, hypertension, and sympathetic overactivity. J Am Soc Hypertens. 2009;3:353-65.

7. Mahfoud F, Schlaich M, Kindermann I, Ukena C, Cremers B, Brandt $\mathrm{MC}$, et al. Effect of renal sympathetic denervation on glucose metabolism in patients with resistant hypertension: a pilot study. Circulation. 2011;123:1940-6.

8. Schlaich MP, Hering D, Sobotka P, Krum H, Lambert GW, Lambert E, et al. Effects of renal denervation on sympathetic activation, blood pressure, and glucose metabolism in patients with resistant hypertension. Front Physiol. 2012;3:10.

9. Witkowski A, Prejbisz A, Florczak E, Kądziela J, Śliwiński P, Bieleń $\mathrm{P}$, et al. Effects of renal sympathetic denervation on blood pressure, 
sleep apnea course, and glycemic control in patients with resistant hypertension and sleep apnea. Hypertension. 2011;58:559-65.

10. Bousquet P, Feldman J, Schwartz J. Central cardiovascular effects of alpha adrenergic drugs: differences between catecholamines and imidazolines. J Pharmacol Exp Ther. 1984;230:232-6.

11. Schann S, Bruban V, Pompermayer K, Feldman J, Pfeiffer B, Renard P, et al. Synthesis and biological evaluation of pyrrolinic isosteres of rilmenidine. Discovery of cis-/trans-dicyclopropylmethyl-(4,5-dimethyl-4,5-dihydro-3H-pyrrol-2-yl)-amine (LNP 509), an I1 imidazoline receptor selective ligand with hypotensive activity. J Med Chem. 2001;44:1588-93.

12. Bousquet P, Ehrhardt JD, Fellmann L, Gasparik V, Greney H, Hadjeri $\mathrm{M}$, et al. Novel amino-pyrroline derivatives, and use thereof in the prevention and/or treatment of metabolic syndrome. WO 2012/143660 A1, 2011 [patent].

13. Gasparik V, Greney H, Schann S, Feldman J, Fellmann L, Ehrhardt JD, et al. Synthesis and biological evaluation of 2aryliminopyrrolidines as selective ligands for I1 imidazoline receptors: discovery of new sympatho-inhibitory hypotensive agents with potential beneficial effects in metabolic syndrome. J Med Chem. 2015;58:878-87.

14. Fellmann L, Regnault V, Greney H, Gasparik V, Muscat A, Max JP, et al. A new pyrroline compound selective for i1-imidazoline receptors improves metabolic syndrome in rats. J Pharmacol Exp Ther. 2013;346:370-80.

15. Weiss M, Bouchoucha S, Aiad F, Ayme-Dietrich E, Dali-Youcef $\mathrm{N}$, Bousquet $\mathrm{P}$, et al. Imidazoline-like drugs improve insulin sensitivity through peripheral stimulation of adiponectin and AMPK pathways in a rat model of glucose intolerance. Am J Physiol Endocrinol Metab. 2015;309:E95-104.

16. Shehzad A, Iqbal W, Shehzad O, Lee YS. Adiponectin: regulation of its production and its role in human diseases. Hormones (Athens). 2012;11:8-20

17. Whitehead JP, Richards AA, Hickman IJ, Macdonald GA, Prins JB. Adiponectin-a key adipokine in the metabolic syndrome. Diabetes Obes Metab. 2006;8:264-80.

18. Kadowaki T, Yamauchi T. Adiponectin and adiponectin receptors. Endocr Rev. 2005;26:439-51.

19. Ruderman NB, Carling D, Prentki M, Cacicedo JM. AMPK, insulin resistance, and the metabolic syndrome. J Clin Invest. 2013;123:2764-72.

20. Hasenour CM, Berglund ED, Wasserman DH. Emerging role of AMP-activated protein kinase in endocrine control of metabolism in the liver. Mol Cell Endocrinol. 2013;366:152-62.

21. Luo L, Liu M. Adipose tissue in control of metabolism. J Endocrinol. 2016;231:R77-R99.

22. Gómez-Hernández A, Beneit N, Díaz-Castroverde S, Escribano Ó. Differential role of adipose tissues in obesity and related metabolic and vascular complications. Int J Endocrinol. 2016;2016:1216783.

23. Bae YJ, Kim SH, Chung JH, Song SW, Kim KS, Kim MK, et al. Evaluation of adiposity-related biomarkers as metabolic syndrome indicators. Clin Nutr Res. 2013;2:91-9.

24. Aubertin G, Sayeh A, Dillenseger JP, Ayme-Dietrich E, Choquet $\mathrm{P}$, Niederhoffer N. Comparison of bioimpedance spectroscopy and $\mathrm{X}$-Ray micro-computed tomography for total fat volume measurement in mice. PLoS ONE. 2017;12:e0183523.

25. Erdös B, Snipes JA, Miller AW, Busija DW. Cerebrovascular dysfunction in Zucker obese rats is mediated by oxidative stress and protein kinase C. Diabetes. 2004;53:1352-9.

26. Oltman CL, Richou LL, Davidson EP, Coppey LJ, Lund DD, Yorek MA. Progression of coronary and mesenteric vascular dysfunction in Zucker obese and Zucker diabetic fatty rats. Am J Physiol Heart Circ Physiol. 2006;291:H1780-7.

27. Després JP, Lemieux I, Bergeron J, Pibarot P, Mathieu P, Larose $\mathrm{E}$, et al. Abdominal obesity and the metabolic syndrome: contribution to global cardiometabolic risk. Arterioscler Thromb Vasc Biol. 2008;28:1039-49.

28. Lambert E, Straznicky NE, Dawood T, Ika-Sari C, Grima M, Esler $\mathrm{MD}$, et al. Change in sympathetic nerve firing pattern associated with dietary weight loss in the metabolic syndrome. Front Physiol. 2011;2:52.

29. Straznicky NE, Lambert GW, McGrane MT, Masuo K, Dawood T, Nestel PJ, et al. Weight loss may reverse blunted sympathetic neural responsiveness to glucose ingestion in obese subjects with metabolic syndrome. Diabetes. 2009;58: 1126-32.

30. Skrapari I, Tentolouris N, Katsilambros N. Baroreflex function: determinants in healthy subjects and disturbances in diabetes, obesity and metabolic syndrome. Curr Diabetes Rev. 2006;2:329-38.

31. Huber DA, Schreihofer AM. Attenuated baroreflex control of sympathetic nerve activity in obese Zucker rats by central mechanisms. J Physiol. 2010;588(Pt 9):1515-25.

32. Straznicky NE, Lambert GW, Masuo K, Dawood T, Eikelis N, Nestel PJ, et al. Blunted sympathetic neural response to oral glucose in obese subjects with the insulin-resistant metabolic syndrome. Am J Clin Nutr. 2009;89:27-36.

33. Lidell ME, Betz MJ, Enerbäck S. Brown adipose tissue and its therapeutic potential. J Intern Med. 2014;276:364-77.

34. Thoonen R, Hindle AG, Scherrer-Crosbie M. Brown adipose tissue: the heat is on the heart. Am J Physiol Heart Circ Physiol. 2016;310:H1592-605.

35. Peterson CM, Orooji M, Johnson DN, Naraghi-Pour M, Ravussin E. Brown adipose tissue does not seem to mediate metabolic adaptation to overfeeding in men. Obesity (Silver Spring). 2017;25:502-5.

36. Pita J, Panadero A, Soriano-Guillén L, Rodríguez E, Rovira A. The insulin sensitizing effects of PPAR- $\gamma$ agonist are associated to changes in adiponectin index and adiponectin receptors in Zucker fatty rats. Regul Pept. 2012;174:18-25.

37. Masaki T, Chiba S, Yasuda T, Tsubone T, Kakuma T, Shimomura I, et al. Peripheral, but not central, administration of adiponectin reduces visceral adiposity and upregulates the expression of uncoupling protein in agouti yellow (Ay/a) obese mice. Diabetes. 2003;52:2266-73

38. Ohno H, Shinoda K, Spiegelman BM, Kajimura S. PPAR $\gamma$ agonists induce a white-to-brown fat conversion through stabilization of PRDM16 protein. Cell Metab. 2012;15:395-404.

39. Seale P, Bjork B, Yang W, Kajimura S, Chin S, Kuang S, et al. PRDM16 controls a brown fat/skeletal muscle switch. Nature. 2008;454:961-7.

40. Edwards L, Fishman D, Horowitz P, Bourbon N, Kester M, Ernsberger P. The I1-imidazoline receptor in PC12 pheochromocytoma cells activates protein kinases $\mathrm{C}$, extracellular signalregulated kinase (ERK) and c-jun N-terminal kinase (JNK). J Neurochem. 2001;79:931-40.

41. Greney H, Ronde P, Magnier C, Maranca F, Rascente C, Quaglia $\mathrm{W}$, et al. Coupling of $\mathrm{I}(1)$ imidazoline receptors to the cAMP pathway: studies with a highly selective ligand, benazoline. Mol Pharmacol. 2000;57:1142-51.

42. Regunathan S, Evinger MJ, Meeley MP, Reis DJ. Effects of clonidine and other imidazole-receptor binding agents on second messenger systems and calcium influx in bovine adrenal chromaffin cells. Biochem Pharmacol. 1991;42:2011-8.

43. Ernsberger P, Ishizuka T, Liu S, Farrell CJ, Bedol D, Koletsky RJ, et al. Mechanisms of antihyperglycemic effects of moxonidine in the obese spontaneously hypertensive Koletsky rat (SHROB). J Pharmacol Exp Ther. 1999;288:139-47.

44. O'Neill HM, Holloway GP, Steinberg GR. AMPK regulation of fatty acid metabolism and mitochondrial biogenesis: implications for obesity. Mol Cell Endocrinol. 2013;366:135-51. 


\section{Affiliations}

Gaëlle Aubertin ${ }^{1} \cdot$ Maud Weiss $^{1} \cdot$ Florian Traversi $^{1} \cdot$ Djamil Benameur $^{1} \cdot$ Philippe Choquet $^{2,3} \cdot$ Nassim Dali-Youcef ${ }^{4,5}$. Françoise Pons ${ }^{6} \cdot$ Séverine Sigrist $^{7} \cdot$ Hugues Greney ${ }^{1} \cdot$ Laurent Monassier $^{1} \cdot$ Pascal Bousquet $^{1}$. Nathalie Niederhoffer ${ }^{1}$

1 Laboratoire de Pharmacologie et Toxicologie

NeuroCardiovasculaire (LPTNC) - EA7296, Faculté de Médecine, Fédération de Médecine Translationnelle, Université de

Strasbourg, Strasbourg, France

2 Imagerie Préclinique - UF6237, Pôle d'imagerie, Hôpitaux Universitaires, Strasbourg, France

3 Laboratoire des sciences de l'ingénieur, de l'informatique et de l'imagerie (ICube) - UMR 7357, Fédération de Médecine Translationnelle de Strasbourg, Université de Strasbourg, Strasbourg, France

4 Laboratoire de Biochimie et Biologie Moléculaire, Hôpitaux Universitaires, Strasbourg, France
5 Institut de Génétique et Biologie Moléculaire et Cellulaire (IGBMC), CNRS UMR7104/INSERM U964/ Université de Strasbourg, Illkirch, France

6 Conception et Application de Molécules Bioactives (CAMB) UMR7199, Faculté de Pharmacie, Université de Strasbourg, Illkirch, France

7 Diabète et thérapie cellulaire (DIATHEC) - EA7294, Centre Européen d'Étude du Diabète, Université de Strasbourg, Strasbourg, France 